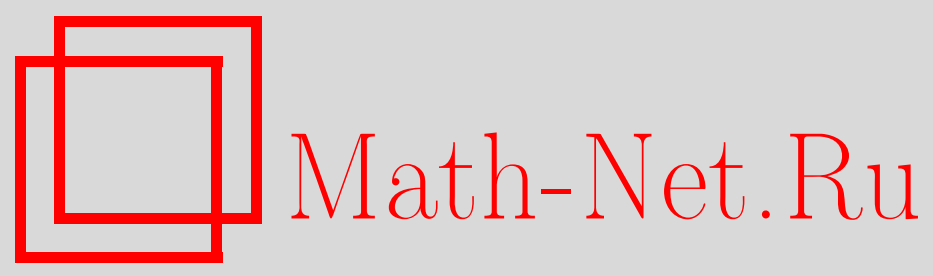

В. В. Гусев, Легальные сообщения для режимов работы блочных шифров ГОСТ Р 34.13-2015, Матем. вопр. криптогр., 2019, том 10, выпуск 3, 33-47

DOI: https://doi.org/10.4213/mvk298

Использование Общероссийского математического портала Math-Net.Ru подразумевает, что вы прочитали и согласны с пользовательским соглашением http://www.mathnet.ru/rus/agreement

Параметры загрузки:

IP : 52.205.19.152

26 апреля 2023 г., 04:28:50

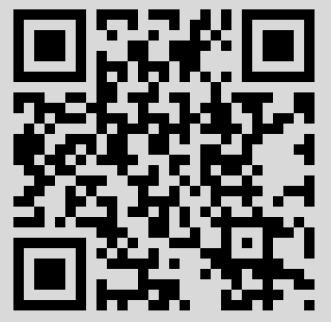


МАТЕМАТИЧЕСКИЕ ВОПРОСЫ КРИПТОГРАФИИ

2019 T. 10 № 3 C. $33-47$

УДК 519.719 .2

DOI https://doi.org/10.4213/mvk298

\title{
Легальные сообщения для режимов работы блочных шифров ГОСТ Р 34.13-2015
}

\author{
В. В. Гусев
}

Технический комитет по стандартизачии (ТK 26), Москва

Получено 18.IV.2019

Аннотация. Для различных режимов блочного шифрования алгоритма ГОСТ Р 34.13-2015 представлены способы формирования сообщений, позволяющие сторонним лицам, не имеющим ключей, навязывать обладателям ключей шифрованные тексты, которые после расшифрования идентифицируются как истинные по имитовставке.

Ключевые слова: легальное сообщение, имитовставка, имитозащита открытых данных, имитозащита шифрованных данных

\section{Legal messages for the GOST R 34.13-2015 block cipher modes}

\section{V. Gusev}

Technical Committee for Standardization TC 26, Moscow

Abstract. For different modes of block enciphering by the GOST R 34.13-2015 algorithm we suggest methods permitting the nonlegal parties which have no information on the secret keys to construct messages which after deciphering will be recognized by the message authentication code as the legal ones.

Keywords: legal message, message authentication code, plaintext authentication, ciphertext authentication 


\section{1. Введение}

ГОСТ Р 34.13-2015 [1] содержит описание шести режимов работы блочного шифрования, которые определяют способы криптографического преобразования открытой информации. Пять из них относятся к шифрованию данных и один - к выработке имитовставки, добавляемой к шифрованному сообщению для обеспечения целостности передаваемой информации. Имитовставка в ГОСТ Р 34.13-2015 представляет собой значение некоторой функции, зависящее от секретного ключа и последовательности данных произвольной длины. Эта функция называется функиией имитозащиты.

В дальнейшем будем рассматривать следующий порядок формирования передаваемого сообщения. Открытая информация $X=\left(x_{0}, x_{1}, \ldots, x_{L-1}\right), \quad$ подлежащая криптографической защите, зашифровывается в одном из режимов работы блочного шифрования на ключе $K$. В режиме выработки имитовставки на ключе $K^{*}$ формируется имитовставка $I M$, которая определенным образом зависит от открытой информации $X$. Передаче подлежит совокупность $(S ; Y ; I M)$, которую в дальнейшем будем называть сообщением. Здесь $S$ - синхропосылка (отсутствует в сообщении, если не используется в режиме шифрования), $Y=\left(y_{0}, y_{1}, \ldots, y_{L-1}\right)$ - шифрованный текст, полученный из $X=\left(x_{0}, x_{1}, \ldots, x_{L-1}\right)$ на ключе $K$.

В настоящее время криптографическая стойкость функции имитозащиты, которая формирует имитовставку, определяется эффективностью решения ряда традиционных задач, связанных с построением прообраза, второго прообраза, коллизии прообразов (см., например, [3,4]). Если такие решения существуют в рамках некоторых требований или условий, то считается, что это определенная слабость функции имитозащиты. При этом, как правило, игнорируются вопросы, связанные с возможностью построения шифрованных текстов, которые соответствуют данным прообразам. Последнее связано с понятием навязывания «ложной» информации, которую обладатель ключей $K, K^{*}$ воспринимает как истинную.

Если придерживаться общепринятой терминологии, то стойкость функции имитозащиты можно оценивать по-другому: эффективностью решения задач, связанных с коллизиями образов. Например, задачу построения второго образа можно сформулировать следующим образом. Без знания ключей $K, K^{*}$ необходимо при известном $(S ; Y ; I M)$ сформировать такое сообщение $(\tilde{S} ; \tilde{Y} ; I \tilde{M})$, что $\tilde{X} \neq X, I M=I \tilde{M}$, где $\tilde{X}, X$ - открытые тексты, полученные в результате расшифрования текстов 
$\tilde{Y}, Y$ соответственно. Стойкость функции имитозащиты можно оценивать как вероятность выполнения условий $\tilde{X} \neq X, I M=I \tilde{M}$.

Насколько известно автору, стойкость функции имитозащиты с таких позиций оценивалась только в [2] (для случая $K=K^{*}$ ).

В данной работе для различных режимов и параметров блочного шифрования алгоритма ГОСТ Р 34.13-2015 решается именно такая задача, связанная с навязыванием «ложной» информации: формирование сообщений (без знания ключей $K, K^{*}$ ), которые при расшифровании в соответствующем режиме и вычислении имитовставки идентифицировались бы пользователем, имеющим ключи, как истинные (целостные), соответствующие некоторым открытым текстам.

Мы будем рассматривать следующий протокол аутентификации сообщения $(S ; Y ; I M)$, который определяется только значением имитовставки $I M$.

Целостность открытых данных $X$, полученных при расшифровании данных $Y$, с помощью имитовставки $I M$ проверяется пользователем, обладающим ключами $K, K^{*}$. Для этого информационные данные $Y$ из сообщения $(S ; Y ; I M)$ расшифровываются на ключе $K$ в соответствующем режиме, и по полученным и принятым данным $X, Y$ на ключе $K^{*}$ вычисляется имитовставка, которая сравнивается с принятой $I M$. Только в случае совпадения имитовставок полученные в результате расшифрования открытые данные $X$ не отвергаются и считаются по протоколу аутентификации истинными (целостными).

Определение. Любое сообщение $(S ; Y ; I M)$ в данном режиме шифрования назовем легальным , если после расшифрования $Y$ в этом режиме на ключе $K$ полученные данные $X$ считаются истинными.

Пользователь, обладающий ключами $K, K^{*}$, всегда формирует легальные сообщения. Пользователь, который не имеет этих ключей, формально не обладает такой возможностью. Но, располагая определенной информацией в виде открытых и шифрованных текстов и имитовставок, он может попытаться сформировать сообщение, которое с определенной вероятностью окажется легальным.

Довольно подробно вопросы, связанные с легальностью сообщений для случая $K^{*}=K$, рассматривались в [2]. Представленные в этой работе способы формирования сообщений (без знания ключа $K$ ) позволяют сторонним лицам навязывать тексты, которые идентифицируются обладателем ключа $K$ после расшифрования как легальные по имитовставке. В [2] показано, что для различных режимов шифрования 
и функций имитозащиты в зависимости от известной исходной информации и ее объема возможна с определенной вероятностью подмена открытого текста, переданного в зашифрованном виде пользователем, имеющим ключ $K$.

В ГОСТ Р 34.13-2015 режимы шифрования и функция имитозащиты реализуются через базовый алгоритм простой замены $E_{K}(x)$, которая представляет собой отображение $V_{N} \times V_{n}$ в $V_{n}\left(K \in V_{N}, x \in V_{n}\right)$ и является подстановкой на $V_{n}$ при любом фиксированном значении $K ; V_{N}, V_{n}$ - множество всех двоичных векторов соответствующей размерности. Здесь $K$ - секретный ключ, $x-n$-битовый блок, который шифруется.

Всегда будем предполагать, что перед выполнением шифрования в любом из режимов открытые данные $X$ разбиваются на блоки размерности $n$ бит в каждом $X=\left(x_{0}, x_{1}, \ldots, x_{L-1}\right), \quad x_{i} \in V_{n}$.

Опишем режим выработки имитовставки в ГОСТ Р 34.13-2015.

\section{Режим выработки имитовставки}

Для обеспечения имитозащиты данных $Z=\left(z_{0}, z_{1}, \ldots, z_{\tilde{L}-1}\right)$ вырабатывается дополнительный блок $I M$ (зависящий от $Z$ и $K^{*}$ ) из $n^{*}$ бит, $1 \leqslant n^{*} \leqslant n, \quad K^{*}$ - секретный ключ имитозащиты. Здесь опишем алгоритм формирования имитовставки для случая, когда $\tilde{L}=L$ и в качестве $Z$ выбирается открытый $Z=X=\left(x_{0}, x_{1}, \ldots, x_{L-1}\right)$ или соответствующий ему шифрованный текст $Z=Y=\left(y_{0}, y_{1}, \ldots, y_{L-1}\right)$.

Этот алгоритм будем называть имитозащитой открытых данных, если $Z=X$, или имитозащитой шифрованных данных, если $Z=Y$.

Пусть $X=\left(x_{0}, x_{1}, \ldots, x_{L-1}\right)$ - открытые данные. Сначала итеративно формируется последовательность $R_{i}$, которую будем называть $n p o$ межуточными значениями функиии имитозащиты,

$$
R_{i}=E_{K^{*}}\left(x_{i} \oplus R_{i-1}\right), i=\overline{0, L-2}, \quad R_{L-1}=E_{K^{*}}\left(x_{L-1} \oplus R_{L-2} \oplus \xi\right),
$$

где $R_{-1}=0$, а $\xi$ известным образом зависит от $E_{K^{*}}(0)$.

Имитовставка $I M$ определяется равенством $I M=f\left(R_{L-1}\right)$, где $f-$ известная функция $f: V_{n} \rightarrow V_{n^{*}}$.

Для случая когда $Z=Y=\left(y_{0}, y_{1}, \ldots, y_{L-1}\right)$, имитовставка вычисляется так же, но в формулах (1) переменные $x_{i}$ заменяются на $y_{i}$.

\section{2. Имитозащита открытых данных}

Для различных режимов шифрования ГОСТ Р 34.13-2015 в пунктах, относящихся к каждому из режимов, будет изложено однотипное 
описание процедуры формирования легальных сообщений. При рассмотрении каждого режима будем иметь в виду следующее.

1. Лицо, формирующее сообщение $(\tilde{S} ; \tilde{Y} ; I \tilde{M})$, не имеет ключей $K, K^{*}$. Компоненты текста $\tilde{Y}$ известны. Они формируются из известной открытой информации, которая доступна и состоит из синхропосылок, открытых и шифрованных текстов, перечисленных в постановке задачи в начале соответствующего пункта.

2. Расшифровывает текст $\tilde{Y}$ и вычисляет имитовставку от полученного текста $\tilde{X}$ пользователь, обладающий ключами $K, K^{*}$.

3. Отличие текста $\tilde{X}$, полученного после расшифрования $\tilde{Y}$ из легального сообщения $(\tilde{S} ; \tilde{Y} ; I \tilde{M})$, от известных открытых текстов, перечисленных в постановке задачи, как правило, будет следовать из того, что они разной длины.

4. В ГОСТ Р 34.13-2015 взаимосвязь между ключом $K$, предназначенным для шифрования, и ключом $K^{*}$ имитозащиты формально не определена. Существует только рекомендация не использовать одинаковые ключи в этих режимах.

В начале каждого пункта, относящегося к данному режиму шифрования, излагается способ построения легального сообщения, относящийся только к случаю $K^{*}=K$. Если, на наш взгляд, существует содержательный вариант построения легального сообщения для $K^{*} \neq K$, то он приводится в конце пункта.

5. Практически во всех случаях при построении легальных сообщений будет использован один и тот же стандартный прием. Легальность сообщения будет следовать из очевидного утверждения: имитовставки текстов

$$
X=\left(x_{0}, x_{1}, \ldots, x_{L-1}\right) \text { и } \tilde{X}=\left(b_{0}, x_{0} \oplus E_{\tilde{K}}\left(b_{0}\right), x_{1}, \ldots, x_{L-1}\right),
$$

вычисленные на ключе $\tilde{K}$, совпадают.

6. Для случая когда $K^{*} \neq K$, варианты легальных сообщений приведены в предположении, что для некоторого известного $b_{0}$ известно значение $E_{K^{*}}\left(b_{0}\right)$.

\section{1. Режим простой замены}

Результатом зашифрования текста $X=\left(x_{0}, x_{1}, \ldots, x_{L-1}\right)$ на ключе $K$ является последовательность $Y=\left(y_{0}, y_{1}, \ldots, y_{L-1}\right), \quad$ где $y_{i}=E_{K}\left(x_{i}\right), \quad i=\overline{0, L-1}$.

Для расшифрования данных $Y=\left(y_{0}, y_{1}, \ldots, y_{L-1}\right)$ используется преобразование $E_{K}^{-1}: \quad x_{i}=E_{K}^{-1}\left(y_{i}\right), \quad i=\overline{0, L-1}$. 
Примеры построения сообщений для режима простой замены и вероятности их легальности приведены и достаточно подробно описаны B [2].

\section{2. Режим гаммирования}

В режиме гаммирования вырабатывается гамма наложения $\gamma_{i}(i=\overline{0, L-1})$, которая зависит от синхропосылки $s_{0}$ :

$$
\gamma_{0}=E_{K}\left(s_{0}\right) ; \quad \gamma_{i}=E_{K}\left(s_{i}\right), \quad s_{i}=\left(s_{i-1}+1\right) \quad \bmod 2^{n}, \quad i=\overline{1, L-1} .
$$

Результатом зашифрования $X=\left(x_{0}, x_{1}, \ldots, x_{L-1}\right) \quad$ на $\quad$ ключе $K$ является последовательность $Y=\left(y_{0}, y_{1}, \ldots, y_{L-1}\right), \quad$ где $y_{i}=x_{i} \oplus \gamma_{i}, \quad i=\overline{0, L-1}$.

Для расшифрования данных $Y=\left(y_{0}, y_{1}, \ldots, y_{L-1}\right) \quad$ по синхропосылке $s_{0}$ по формуле (2) вычисляются $\gamma_{i}$, и тогда $x_{i}=y_{i} \oplus \gamma_{i}, \quad i=\overline{0, L-1}$.

Пусть известны два легальных сообщения $\left(s_{0} ; Y ; I M\right), \quad\left(s_{0}^{*} ; Y^{*} ; I M^{*}\right)$ и открытые тексты $X=\left(x_{0}, x_{1}, \ldots, x_{L-1}\right)$, $X^{*}=\left(x_{0}^{*}, x_{1}^{*}, \ldots, x_{L^{*}-1}^{*}\right), \quad$ соответствующие шифрованным текстам $Y=\left(y_{0}, y_{1}, \ldots, y_{L-1}\right), \quad Y^{*}=\left(y_{0}^{*}, y_{1}^{*}, \ldots, y_{L^{*}-1}^{*}\right), \quad$ где $L^{*} \geqslant L+1$, $\gamma_{i}=E_{K}\left(s_{i}\right), \quad \gamma_{i}^{*}=E_{K}\left(s_{i}^{*}\right)$.

Формируется сообщение $\left(s_{0}^{*} ; \tilde{Y} ; I M\right)$ :

$$
\tilde{Y}=\left(s_{i_{0}} \oplus \gamma_{0}^{*}, x_{0} \oplus \gamma_{i_{0}} \oplus \gamma_{1}^{*}, x_{1} \oplus \gamma_{2}^{*}, \ldots, x_{L-1} \oplus \gamma_{L}^{*}\right), \quad 0 \leqslant i_{0} \leqslant L-1 .
$$

При расшифровании получается текст $\tilde{X}=\left(s_{i_{0}}, x_{0} \oplus \gamma_{i_{0}}, x_{1}, \ldots, x_{L-1}\right)$, в котором $\gamma_{i_{0}}=E_{K}\left(s_{i_{0}}\right)$, и поэтому сообщение $\left(s_{0}^{*} ; \tilde{Y} ; I M\right)$ будет легальным.

Для случая $K^{*} \neq K$ легальным будет сообщение $\left(s_{0}^{*} ; \tilde{Y} ; I M\right)$, в котоpoм $\tilde{Y}=\left(b_{0} \oplus \gamma_{0}^{*}, x_{0} \oplus E_{K^{*}}\left(b_{0}\right) \oplus \gamma_{1}^{*}, x_{1} \oplus \gamma_{2}^{*}, \ldots, x_{L-1} \oplus \gamma_{L}^{*}\right)$. При расшифровании получается текст $\tilde{X}=\left(b_{0}, x_{0} \oplus E_{K^{*}}\left(b_{0}\right), x_{1}, \ldots, x_{L-1}\right)$.

\section{3. Режим гаммирования с обратной связью по выходу}

В этом режиме вырабатывается гамма наложения $\gamma_{i}(i=\overline{0, L-1})$, которая зависит от синхропосылки $S=\left(s_{0}, s_{1}, \ldots, s_{r-1}\right), s_{j} \in V_{n}$. Значение $r \geqslant 1$ является параметром данного режима (в оригинальном тексте [1] этот параметр обозначен буквой «Z»).

Знаки гаммы вырабатываются по следующему правилу:

$$
\gamma_{0}=E_{K}\left(s_{0}\right), \quad \gamma_{1}=E_{K}\left(s_{1}\right), \ldots, \quad \gamma_{r-1}=E_{K}\left(s_{r-1}\right),
$$




$$
\gamma_{i+r}=E_{K}\left(\gamma_{i}\right), \quad i=0,1, \ldots, L-1-r .
$$

Результатом зашифрования текста $X=\left(x_{0}, x_{1}, \ldots, x_{L-1}\right)$ на ключе $K$ является последовательность $Y=\left(y_{0}, y_{1}, \ldots, y_{L-1}\right)$, где $y_{i}=x_{i} \oplus \gamma_{i}, \quad i=\overline{0, L-1}$. Для расшифрования используется обратное преобразование.

Рассмотрим два случая.

3.1. $r=1$.

Пусть известны два легальных сообщения $\left(s_{0} ; Y ; I M\right),\left(s_{0}^{*} ; Y^{*} ; I M^{*}\right)$ и открытые тексты $X=\left(x_{0}, x_{1}, \ldots, x_{L-1}\right), \quad X^{*}=\left(x_{0}^{*}, x_{1}^{*}, \ldots, x_{L^{*}-1}^{*}\right)$, соответствующие шифрованным текстам $Y=\left(y_{0}, y_{1}, \ldots, y_{L-1}\right)$, $Y^{*}=\left(y_{0}^{*}, y_{1}^{*}, \ldots, y_{L^{*}-1}^{*}\right)$, и $L^{*} \geqslant L+1$. Знаки гаммы $\gamma_{i}$ и $\gamma_{i}^{*}$ вычислены по формулам (3).

Сообщение $\left(s_{0}^{*} ; \tilde{Y} ; I M\right)$, где

$$
\tilde{Y}=\left(\gamma_{i_{0}} \oplus \gamma_{0}^{*}, x_{0} \oplus \gamma_{i_{0}+1} \oplus \gamma_{1}^{*}, x_{1} \oplus \gamma_{2}^{*}, \ldots, x_{L-1} \oplus \gamma_{L}^{*}\right), \quad 0 \leqslant i_{0} \leqslant L-2,
$$

является легальным при $L \geqslant 2$.

При расшифровании получается текст $\tilde{X}=\left(\gamma_{i_{0}}, x_{0} \oplus \gamma_{i_{0}+1}, x_{1}\right.$, $\left.\ldots, x_{L-1}\right)$, в котором $\gamma_{i_{0}+1}=E_{K}\left(\gamma_{i_{0}}\right)$.

Для случая $K^{*} \neq K$ легальным будет сообщение с текстом $\tilde{Y}=\left(b_{0} \oplus \gamma_{0}^{*}, x_{0} \oplus E_{K^{*}}\left(b_{0}\right) \oplus \gamma_{1}^{*}, x_{1} \oplus \gamma_{2}^{*}, \ldots, x_{L-1} \oplus \gamma_{L}^{*}\right)$.

3.2. $r \geqslant 2$.

Пусть известны легальное сообщение $(S ; Y ; I M)$ и открытый текст $X=\left(x_{0}, x_{1}, \ldots, x_{L-1}\right)$, из которого оно получено на синхропосылке $S=\left(s_{0}, s_{1}, \ldots, s_{r-1}\right)$. Пусть $L \geqslant r+1$ и $L$ не делится на $r$.

Формируется сообщение $(\tilde{S} ; \tilde{Y} ; I M)$, где $\tilde{S}=\left(s_{0}, s_{0}, s_{1}, \ldots, s_{r-2}\right)$. Гамма наложения $\left(\tilde{\gamma}_{0}, \tilde{\gamma}_{1}, \ldots, \tilde{\gamma}_{L}\right)$ длины $(L+1)$ вырабатывается из $\tilde{S}$ в соответствии с (3), шифрованный текст $\tilde{Y}=\left(s_{i_{0}} \oplus \tilde{\gamma}_{0}, x_{0} \oplus \gamma_{i_{0}} \oplus \tilde{\gamma}_{1}, x_{1} \oplus \tilde{\gamma}_{2}, \ldots, x_{L-1} \oplus \tilde{\gamma}_{L}\right) \quad\left(0 \leqslant i_{0} \leqslant r-1\right)$. Такую гамму наложения можно сформировать, так как $L$ не делится на $r$.

При расшифровании получается текст $\tilde{X}=\left(s_{i_{0}}, x_{0} \oplus \gamma_{i_{0}}, x_{1}, \ldots, x_{L-1}\right)$, в котором $\gamma_{i_{0}}=E_{K}\left(s_{i_{0}}\right)$.

Для случая $K^{*} \neq K$ легальным будет сообщение, в котором $\tilde{Y}=\left(b_{0} \oplus \tilde{\gamma}_{0}, x_{0} \oplus E_{K^{*}}\left(b_{0}\right) \oplus \tilde{\gamma}_{1}, x_{1} \oplus \tilde{\gamma}_{2}, \ldots, x_{L-1} \oplus \tilde{\gamma}_{L}\right)$.

\section{4. Режим простой замены с зацеплением}

Этот режим шифрования зависит от синхропосылки $S=\left(s_{0}, s_{1}, \ldots, s_{r-1}\right), s_{j} \in V_{n}$. Значение $r \geqslant 1$ является параметром 
данного режима (в оригинальном тексте ГОСТ Р 34.13-2015 [1] этот параметр обозначен буквой «Z»).

Шифрование открытого текста $X=\left(x_{0}, x_{1}, \ldots, x_{L-1}\right)$ осуществляется следующим образом:

$$
\begin{aligned}
& y_{0}=E_{K}\left(s_{0} \oplus x_{0}\right), y_{1}=E_{K}\left(s_{1} \oplus x_{1}\right), \ldots, y_{r-1}=E_{K}\left(s_{r-1} \oplus x_{r-1}\right), \\
& y_{i+r}=E_{K}\left(y_{i} \oplus x_{i+r}\right), i=0,1, \ldots, L-1-r .
\end{aligned}
$$

Для расшифрования текста $Y=\left(y_{0}, y_{1}, \ldots, y_{L-1}\right)$ используются соотношения

$$
\begin{aligned}
& x_{0}=E_{K}^{-1}\left(y_{0}\right) \oplus s_{0}, x_{1}=E_{K}^{-1}\left(y_{1}\right) \oplus s_{1}, \ldots, x_{r-1}=E_{K}^{-1}\left(y_{r-1}\right) \oplus s_{r-1}, \\
& x_{i+r}=E_{K}^{-1}\left(y_{i+r}\right) \oplus y_{i}, \quad i=0,1, \ldots, L-1-r .
\end{aligned}
$$

Рассмотрим два случая.

4.1. $r=1$.

Пусть известны легальное сообщение $\left(s_{0} ; Y ; I M\right)$ и открытый текст $X=\left(x_{0}, x_{1}, \ldots, x_{L-1}\right)$, из которого оно получено на синхропосылке $s_{0}$. При $r=1$ тексты $X$ и $Y$ связаны соотношением

$$
y_{i}=E_{K}\left(y_{i-1} \oplus x_{i}\right), i=0,1, \ldots, L-1, \quad y_{-1}=s_{0} .
$$

Положим $Z^{(0)}=\left\{z_{0}, z_{1}, \ldots, z_{L-1}\right\}$, где $z_{i}=y_{i-1} \oplus x_{i}$, и предположим для простоты, что все $z_{i}$ различны. Пусть существуют такие $\tilde{z}, z \in Z^{(0)}$, что

$$
E_{K}(\tilde{z}) \oplus E_{K}(z)=s_{0} .
$$

Тогда сообщение $\left(z^{(0)} ; \tilde{Y} ; I M\right)$, где

$$
z^{(0)}=\tilde{z} \oplus z, \quad \tilde{Y}=\left(E_{K}(\tilde{z}), y_{0}, y_{1}, \ldots, y_{L-1}\right),
$$

является легальным.

При расшифровании получится текст $\tilde{X}=\left(z, x_{0} \oplus E_{K}(z), x_{1}, \ldots, x_{L-1}\right)$.

Можно считать, что при $s_{0} \neq 0$ некоторым приближением вероятности выполнения события (4), т. е. вероятности того, что можно сформировать легальное сообщение указанного вида, является значение $p_{1}=1-\left(1-\frac{1}{2^{n}}\right)^{\frac{L(L-1)}{2}}$.

Для данного режима укажем еще два отличающихся от приведенного выше примера построения легальных сообщений при некоторых дополнительны условиях.

Первый пример. Если $s_{0}=0$, то выполнено условие (4). В этом случае вообще не обязательно знать открытый текст. Легальным будет сообщение $(0 ; \tilde{Y} ; I M)$, где $\tilde{Y}=(\underbrace{y_{0}, \ldots, y_{0}}_{t}, y_{1}, \ldots, y_{L-1}) \quad(t \geqslant 2)$. 
Так как $y_{0}=E_{K}\left(x_{0}\right)$ при $s_{0}=0$, то после расшифрования получается текст

$$
\tilde{X}=(x_{0}, \underbrace{x_{0} \oplus E_{K}\left(x_{0}\right), \ldots, x_{0} \oplus E_{K}\left(x_{0}\right)}_{t-1}, x_{1}, \ldots, x_{L-1}),
$$

для которого промежуточные значения функции имитозащиты в соответствии с (1) равны

$$
\tilde{R}_{0}=\tilde{R}_{1}=\ldots=\tilde{R}_{t-1}=E_{K}\left(x_{0}\right)=R_{0}, \tilde{R}_{t}=R_{1}, \ldots, \tilde{R}_{L-1+t-1}=R_{L-1},
$$

и имитовставка равна $I M$.

Bторой пример. Пусть $x_{0} \in Z^{(0)}\left(Z^{(0)}\right.$ определено в начале данного подпункта), тогда $E_{K}\left(x_{0}\right)$ известно. Легальным будет сообщение $(s ; \tilde{Y} ; I M), \quad$ где $s=E_{K}\left(x_{0}\right) \oplus y_{0}, \tilde{Y}=\left(y_{1}, \ldots, y_{L-1}\right)$. После расшифрования получается текст $\tilde{X}=\left(E_{K}\left(x_{0}\right) \oplus x_{1}, x_{2}, \ldots, x_{L-1}\right)$, имитовставка которого равна $I M$.

Вероятность $p_{1}$ построения легального сообщения, определяемого условием (4), увеличится, если на одном ключе $K$ сформировано несколько легальных сообщений с суммарным числом зашифрованных блоков $L$.

Пусть известны легальные сообщения $\left(s_{0}^{(j)} ; Y^{(j)} ; I M^{(j)}\right)$ и открытые тексты $X^{(j)}=\left(x_{0}^{(j)}, x_{1}^{(j)}, \ldots, x_{L_{0}-1}^{(j)}\right)$, из которых они получены на синхропосылках $s_{0}^{(j)}, \quad j=0,1, \ldots, t-1, \quad L=t L_{0}$.

Так же, как и в начале подпункта 4.1, обозначим через $Z^{(0)}=\left\{z_{0}, z_{1}, \ldots, z_{L-1}\right\}$ совокупность всех $t L_{0}$ значений $y_{i-1}^{(j)} \oplus x_{i}^{(j)}$, и предположим для простоты, что все $z_{i}$ различны. Пусть также все $s_{0}^{(j)}(j=0,1, \ldots, t-1)$ различны и не равны нулю.

Аналогом условия (4) будет выполнение равенства $E_{K}(\tilde{z}) \oplus E_{K}(z)=s_{0}^{(j)}$ для некоторых $0 \leqslant j \leqslant t-1$ и $\tilde{z}, z \in Z^{(0)}$.

Сообщение $\left(z^{(0)} ; \tilde{Y} ; I M^{(j)}\right)$, где

$$
z^{(0)}=\tilde{z} \oplus z, \quad \tilde{Y}=\left(E_{K}(\tilde{z}), y_{0}^{(j)}, y_{1}^{(j)}, \ldots, y_{L_{0}-1}^{(j)}\right),
$$

будет легальным.

При расшифровании получится текст

$$
\tilde{X}=\left(z, x_{0}^{(j)} \oplus E_{K}(z), x_{1}^{(j)}, \ldots, x_{L_{0}-1}^{(j)}\right) .
$$

Вероятность выполнения условия $E_{K}(\tilde{z}) \oplus E_{K}(z)=s_{0}^{(j)}$ можно считать равной $p_{1}=1-\left(1-\frac{t}{2^{n}}\right)^{\frac{L(L-1)}{2}}$. 
Для случая $K^{*} \neq K$ легальное сообщение можно построить, если аналогично (4) существует такое $\tilde{z} \in Z^{(0)}$, что $E_{K}(\tilde{z}) \oplus E_{K^{*}}\left(b_{0}\right)=s_{0}$.

Сообщение $\left(z^{(0)} ; \tilde{Y} ; I M\right)$ будет легальным, если $z^{(0)}=\tilde{z} \oplus b_{0}$, $\tilde{Y}=\left(E_{K}(\tilde{z}), y_{0}, y_{1}, \ldots, y_{L-1}\right)$. При расшифровании $\tilde{Y}$ получаем текст $\tilde{X}=\left(b_{0}, x_{0} \oplus E_{K^{*}}\left(b_{0}\right), x_{1}, \ldots, x_{L-1}\right)$. Вероятность легальности равна $p_{1}=1-\left(1-\frac{1}{2^{n}}\right)^{L}$.

4.2. $r \geqslant 2$.

Пусть известны легальное сообщение $(S ; Y ; I M)$ и открытый текст $X=\left(x_{0}, x_{1}, \ldots, x_{L-1}\right)$, из которого оно получено на синхропосылке $S=\left(s_{0}, \ldots, s_{r-1}\right)$, и $L \geqslant 2$.

Положим, как и в начале пп. 4.1, $Z^{(0)}=\left\{z_{0}, z_{1}, \ldots, z_{L-1}\right\}, \quad$ где $z_{i}=E_{K}^{-1}\left(y_{i}\right), \quad$ и предположим для простоты, что все $z_{i}$ различны.

Приведем два варианта построения легальных сообщений, если выполнены определенные условия.

Пусть $x_{0} \in Z^{(0)}$, т. е. $E_{K}\left(x_{0}\right)$ известно. Сформируем синхропосылку $\tilde{S}=\left(\tilde{s}_{0}, \tilde{s}_{1}, s_{2}, \ldots, s_{r-1}\right)$, которая отличается от $S$ первыми двумя компонентами. Для этого выберем произвольные $z^{(0)} \in Z^{(0)}$ (отличное от $\left.x_{0}\right)$ и $z^{(1)}$ так, чтобы $E_{K}\left(z^{(0)}\right) \oplus z^{(1)}=E_{K}\left(x_{0}\right) \oplus x_{1}$. Положим $\tilde{s}_{0}=s_{0} \oplus x_{0} \oplus z^{(0)}, \quad \tilde{s}_{1}=s_{1} \oplus x_{1} \oplus z^{(1)}$.

Тогда легальным будет сообщение $(\tilde{S} ; Y ; I M)$. При расшифровании получается текст $\tilde{X}=\left(\tilde{x}_{0}, \tilde{x}_{1}, \ldots, \tilde{x}_{L-1}\right)$, где

$$
\begin{aligned}
& \tilde{x}_{0}=E_{K}^{-1}\left(y_{0}\right) \oplus \tilde{s}_{0}=x_{0} \oplus s_{0} \oplus\left(s_{0} \oplus x_{0} \oplus z^{(0)}\right)=z^{(0)}, \\
& \tilde{x}_{1}=E_{K}^{-1}\left(y_{1}\right) \oplus \tilde{s}_{1}=z^{(1)} \\
& \tilde{x}_{2}=E_{K}^{-1}\left(y_{2}\right) \oplus s_{2}=x_{2}, \\
& \tilde{x}_{3}=x_{3}, \ldots, \tilde{x}_{L-1}=x_{L-1} .
\end{aligned}
$$

В силу равенства $E_{K}\left(z^{(0)}\right) \oplus z^{(1)}=E_{K}\left(x_{0}\right) \oplus x_{1}$ имитовставка равна $I M$.

Второй вариант для $r=2$. Пусть существует такое $z^{*} \in Z^{(0)}$, что $E_{K}\left(z^{*}\right)=s_{1}$. Выберем произвольное $z \in Z^{(0)}$ и сформируем сообщение $(\tilde{S} ; \tilde{Y} ; I M)$, где

$$
\left(\tilde{s}_{0}, \tilde{s}_{1}\right)=\left(z^{*} \oplus z, E_{K}(z) \oplus s_{0}\right), \quad \tilde{Y}=\left(s_{1}, y_{0}, y_{1}, \ldots, y_{L-1}\right) .
$$

При расшифровании получается текст $\tilde{X}=\left(\tilde{x}_{0}, \tilde{x}_{1}, x_{1}, \ldots, x_{L-1}\right)$, где $\tilde{x}_{0}=E_{K}^{-1}\left(s_{1}\right) \oplus z^{*} \oplus z=z, \quad \tilde{x}_{1}=E_{K}^{-1}\left(y_{0}\right) \oplus E_{K}(z) \oplus s_{0}=E_{K}(z) \oplus x_{0}$.

В тексте $\tilde{X}$ имитовставка равна $I M$. 
5. Режим гаммирования с обратной связью по шифртексту

Этот режим шифрования зависит от синхропосылки $S=\left(s_{0}, s_{1}, \ldots, s_{r-1}\right), \quad s_{j} \in V_{n}$. Значение $r \geqslant 1$ является параметром данного режима (в оригинальном тексте ГОСТ Р 34.13-2015 [1] этот параметр определяется через «т»и «n», если $m=r n)$.

Шифрование открытого текста $X=\left(x_{0}, x_{1}, \ldots, x_{L-1}\right)$ осуществляется следующим образом:

$$
\begin{aligned}
& y_{0}=E_{K}\left(s_{0}\right) \oplus x_{0}, \quad y_{1}=E_{K}\left(s_{1}\right) \oplus x_{1}, \ldots, y_{r-1}=E_{K}\left(s_{r-1}\right) \oplus x_{r-1}, \\
& y_{i+r}=E_{K}\left(y_{i}\right) \oplus x_{i+r}, \quad i=0,1, \ldots, L-1-r .
\end{aligned}
$$

Для расшифрования текста $Y=\left(y_{0}, y_{1}, \ldots, y_{L-1}\right)$ используются соотношения

$$
\begin{aligned}
& \left.x_{0}=E_{K}\left(s_{0}\right) \oplus y_{0}, \quad x_{1}=E_{K}\left(s_{1}\right) \oplus y_{1}, \ldots, x_{r-1}=E_{K}\left(s_{r-1}\right) \oplus y_{r-1}\right), \\
& x_{i+r}=E_{K}\left(y_{i}\right) \oplus y_{i+r}, \quad i=0,1, \ldots, L-1-r .
\end{aligned}
$$

Рассмотрим два случая.

5.1. $r=1$.

Пусть известны легальное сообщение $\left(s_{0} ; Y ; I M\right)$ и открытый текст $X=\left(x_{0}, x_{1}, \ldots, x_{L-1}\right)$, из которого оно получено на синхропосылке $s_{0}$, $L \geqslant 2$. Тексты $X$ и $Y$ связаны соотношением

$$
y_{i}=E_{K}\left(y_{i-1}\right) \oplus x_{i}, i=0,1, \ldots, L-1, \quad y_{-1}=s_{0} .
$$

Пример построения легального сообщения описан в [2]. Оно имеет вид $\left(s_{0} ;\left(x_{0}, y_{1}, y_{2}, \ldots, y_{L-1}\right) ; I M\right)$, если $E_{K}\left(s_{0}\right) \neq 0, \quad$ и вид $\left(s_{0} ;\left(s_{0}, y_{0}, y_{1}, y_{2}, \ldots, y_{L-1}\right) ; I M\right)$, если $E_{K}\left(s_{0}\right)=0$. При этом достаточно знать только один блок $x_{0}$ в тексте $X$.

В этом подпункте остановимся на возможности построения сообщений, которые можно назвать условно легальными. Смысл такого названия заключается в следующем. Тексты, представленные ниже, будут зависеть от $X, Y$ и неизвестных, вообще говоря, промежуточных значений $R_{i}, \quad i=\overline{0, L-2}$, функции имитозащиты $(1)$, и поэтому формально лицо, не обладающее ключом $K$, не может сформировать из открытых данных $s_{0}, X, Y$ подходящий шифртекст. Но если все значения $R_{0}, R_{1}, \ldots, R_{j-1}$ для текста $X=\left(x_{0}, x_{1}, \ldots, x_{L-1}\right)$ при некотором $j \leqslant L-2$ попадают в совокупность $\left\{y_{-1}, y_{0}, \ldots, y_{L-2}\right\}$, то условно легальные сообщения становятся легальными.

Обозначим через $\vec{R}=\left(R_{0}, R_{1}, \ldots, R_{L-1}\right) \quad$ последовательность промежуточных значений функции имитозащиты (1) для текста $X=\left(x_{0}, x_{1}, \ldots, x_{L-1}\right)$, где, напомним, $R_{i}=E_{K}\left(x_{i} \oplus R_{i-1}\right)$, 
$i=\overline{0, L-2}, \quad R_{L-1}=E_{K}\left(x_{L-1} \oplus R_{L-2} \oplus \xi\right) . \quad$ Шифрованный $\quad$ текст $Y=\left(y_{0}, y_{1}, \ldots, y_{L-1}\right)$ получен в соответствии с $(5)$.

Условно легальным будет сообщение $\left(s_{0} ; \tilde{Y} ; I M\right)$, где $I M-$ имитовставка, вычисленная для текста $X$,

$$
\tilde{Y}=\left(y_{0}, \ldots, y_{i-1}, x_{i} \oplus R_{i-1}, y_{i+1}, y_{i+2}, \ldots, y_{L-1}\right), 0 \leqslant i \leqslant L-2 .
$$

Проверим это. При расшифровании получается текст

$$
\tilde{X}=\left(x_{0}, \ldots, x_{i-1}, y_{i} \oplus R_{i-1}, y_{i+1} \oplus R_{i}, x_{i+2}, \ldots, x_{L-1}\right),
$$

в котором последовательность $\vec{R}^{*}$ промежуточных значений функции имитозащиты выглядит следующим образом:

$$
\vec{R}^{*}=\left(R_{0}, \ldots, R_{i-1}, E_{K}\left(y_{i}\right), R_{i+1}, \ldots, R_{L-1}\right) .
$$

Также условно легальным будет сообщение $\left(s_{0} ; \tilde{Y} ; I M\right)$, где

$$
\begin{aligned}
& \tilde{Y}=\left(y_{0}, \ldots, y_{i-1}, x_{i} \oplus R_{i-1}, x_{i+1} \oplus R_{i}, \ldots, x_{j} \oplus R_{j-1}, y_{j+1}, y_{j+2}, \ldots, y_{L-1}\right), \\
& 0 \leqslant i \leqslant L-3, \quad i<j \leqslant L-2 .
\end{aligned}
$$

При расшифровании получается текст

$$
\tilde{X}=\left(x_{0}, \ldots, x_{i-1}, y_{i} \oplus R_{i-1}, x_{i+1}, \ldots, x_{j}, y_{j+1} \oplus R_{j}, x_{j+2}, \ldots, x_{L-1}\right),
$$

в котором последовательность $\vec{R}^{*}$ имеет вид

$$
\vec{R}^{*}=\left(R_{0}, \ldots, R_{i-1}, E_{K}\left(y_{i}\right), E_{K}\left(y_{i+1}\right), \ldots, E_{K}\left(y_{j}\right), R_{j+1}, \ldots, R_{L-1}\right) .
$$

Используя эти два варианта построения условно легальных сообщений, можно сформировать произвольный текст $\tilde{Y}=\left(\tilde{y}_{0}, \tilde{y}_{1}, \ldots, \tilde{y}_{L-2}, y_{L-1}\right) \quad$ с имитовставкой $I M$, где либо $\tilde{y}_{i}=y_{i}$, либо $\tilde{y}_{i}=x_{i} \oplus R_{i-1}, \quad 0 \leqslant i \leqslant L-2$. Общее число таких вариантов условно легальных сообщений, исключая $\tilde{Y}=Y$, равно $2^{L-1}-1$. Все они попарно различны, если $R_{i-1} \neq E_{K}\left(y_{i-1}\right)$ для всех $0 \leqslant i \leqslant L-2$.

Примером того, что все условно легальные сообщения, сконструированные таким образом, становятся легальными, является случай, когда $E_{K}\left(s_{0}\right)=0$. При этом значении $s_{0}$ шифрованный текст $Y=\left(y_{0}, y_{1}, \ldots, y_{L-1}\right)$ из легального сообщения $\left(s_{0} ; Y ; I M\right)$ имеет вид

$$
\left(y_{0}, y_{1}, \ldots, y_{L-1}\right)=\left(x_{0}, x_{1} \oplus R_{0}, \ldots, x_{L-1} \oplus R_{L-2}\right),
$$

где $R_{i}$ из $(1)$, и все $R_{i}(0 \leqslant i \leqslant L-2)$ известны. 
Так как $R_{-1}=E_{K}\left(s_{0}\right)$, то максимально возможное число легальных сообщений с $\tilde{Y} \neq Y$ для этого случая равно, вообще говоря, $2^{L-2}-1$ при $L \geqslant 3$.

5.2. $r \geqslant 2$.

Пусть известны легальное сообщение $(S ; Y ; I M)$ и открытый текст $X=\left(x_{0}, x_{1}, \ldots, x_{L-1}\right)$, из которого оно получено на синхропосылке $S=\left(s_{0}, s_{1}, \ldots, s_{r-1}\right), \quad L \geqslant r+1$.

Обозначим $Y^{(0)}=\left\{s_{0}, s_{1}, \ldots, s_{r-1}, y_{0}, \ldots, y_{L-1-r}\right\}$ и будем считать для простоты, что все элементы $Y^{(0)}$ различны. Предположим, что существует $z^{*} \in Y^{(0)}$, для которого

$$
E_{K}\left(z^{*}\right)=E_{K}\left(s_{r-1}\right) \oplus E_{K}\left(s_{0}\right) .
$$

Для любого $z^{(1)} \in Y^{(0)}$ положим $z^{(0)}=z^{*} \oplus E_{K}\left(z^{(1)}\right)$.

Тогда сообщение $(\tilde{S} ;(\tilde{Y}, Y) ; I M)$ будет легальным, где

$$
\tilde{S}=\left(z^{(1)}, z^{(0)}, s_{1}, \ldots, s_{r-2}\right), \quad \tilde{Y}=\left(z^{*}, s_{1}, s_{2}, \ldots, s_{r-1}\right) .
$$

При расшифровании получается текст $\tilde{X}=\left(X_{0}, X_{1}\right)$, где

$$
\begin{aligned}
& X_{0}=\left(z^{(0)}, s_{1} \oplus E_{K}\left(z^{(0)}\right), s_{2} \oplus E_{K}\left(s_{1}\right), \ldots, s_{r-1} \oplus E_{K}\left(s_{r-2}\right)\right), \\
& X_{1}=\left(x_{0} \oplus E_{K}\left(s_{r-1}\right), x_{1}, \ldots, x_{L-1}\right) .
\end{aligned}
$$

При вычислении промежуточных значений функции имитозащиты и имитовставки получаем

$$
\begin{aligned}
& \tilde{R}_{0}=E_{K}\left(z^{(0)}\right), \tilde{R}_{1}=E_{K}\left(s_{1}\right), \quad \tilde{R}_{2}=E_{K}\left(s_{2}\right), \ldots, \tilde{R}_{r-1}=E_{K}\left(s_{r-1}\right), \\
& \tilde{R}_{r}=R_{0}, \ldots, \tilde{R}_{r+L-1}=R_{L-1}, \quad I \tilde{M}=I M .
\end{aligned}
$$

Вероятность события (6) при условии, что $E_{K}\left(s_{r-1}\right), E_{K}\left(s_{0}\right) \neq 0$, можно считать равной $p_{1}=1-\left(1-\frac{1}{2^{n}}\right)^{L-2}$. Если же $E_{K}\left(s_{r-1}\right)=0$ или $E_{K}\left(s_{0}\right)=0$, то условие (6) выполняется всегда.

\section{3. Имитозащита шифрованных данных}

В ГОСТ Р 34.13-2015 примечание к режиму выработки имитовставки содержит рекомендацию использовать для этого режима ключ, отличный от ключа шифрования $\left(K^{*} \neq K\right)$. Относительно того, какая информация $Z=\left(z_{0}, z_{1}, \ldots, z_{\tilde{L}-1}\right)$ - открытая, шифрованная или другая подлежит имитозащите, ничего не сказано. Поэтому в этом разделе рассмотрим случай, когда в режиме выработки имитовставки осуществляется имитозащита шифрованных данных $Z=Y=\left(y_{0}, y_{1}, \ldots, y_{L-1}\right)$. 
Пусть $(S ; Y ; I M)$ - легальное сообщение в любом режиме шифрования, в котором используется синхропосылка $S=\left(s_{0}, s_{1}, \ldots, s_{r-1}\right)$, $r \geqslant 1 ; Y=\left(y_{0}, y_{1}, \ldots, y_{L-1}\right)$ - шифрованный текст, полученный в этом режиме на ключе $K$ из открытого текста $X=\left(x_{0}, x_{1}, \ldots, x_{L-1}\right) ; I M$ - имитовставка, вычисленная на ключе $K^{*}$ для имитозащиты данных $Y=\left(y_{0}, y_{1}, \ldots, y_{L-1}\right)$.

Так как $I M$ является значением функции, явно зависящей только от $Y=\left(y_{0}, y_{1}, \ldots, y_{L-1}\right)$, то легальным будет любое сообщение $(\tilde{S} ; Y ; I M)$, где $\widetilde{S} \neq S$. При этом, если все элементы $s_{i}$ синхропосылки $S$ используются при шифровании текста $X$, то текст $\tilde{X}$, полученный при расшифровании $Y$ на синхропосылке $\tilde{S}$, будет заведомо отличаться от $X$. Если не все элементы синхропосылки используются $(L<r)$, то $\tilde{X}$ будет отличаться от $X$, если, например, $\tilde{s}_{0} \neq s_{0}$, где $\tilde{s}_{0}-$ первый элемент синхропосылки $\tilde{S}$.

Отсюда следует, что если режим выработки имитовставки в ГОСТ Р 34.13-2015 используется как имитозащита шифрованных данных, то для навязывания «ложной» информации вообще не обязательно знать открытый текст $X$, из которого было сформировано легальное сообщение $(S ; Y ; I M)$.

\section{4. Выводы}

В данной работе для ГОСТ Р 34.13-2015 представлен отличный от общепринятого подход к оценке стойкости функции имитозащиты. Вместо поиска эффективных способов построения коллизий прообразов решается задача построения коллизий образов. Она подразумевает формирование сообщений (без знания ключей), которые при расшифровании в соответствующем режиме идентифицируются по имитовставке пользователем, имеющим ключи, как истинные (целостные), соответствующие некоторым открытым текстам. Такой подход позволяет не только решать в большинстве случаев задачу нахождения в явном виде коллизии прообразов, но и практически реализовывать навязывание «ложной» информации, т. е. такой открытой информации, которую пользователь, имеющий ключи, не передавал в зашифрованном виде.

В случае когда режим выработки имитовставки используется как имитозащита открытых данных, с определенной вероятностью возможна подмена открытых данных, переданных пользователем, который обладает секретными ключами, или формирование легальных сообщений с заданным значением имитовставки. 
Небольшая часть результатов относится к случаю, когда ключ $K$ шифрования не совпадает с ключом $K^{*}$ имитозащиты $\left(K \neq K^{*}\right)$. Они получены в предположении, что для некоторого известного $b_{0}$ известно значение $E_{K^{*}}\left(b_{0}\right)$. Большая часть результатов относится к случаю, когда $K=K^{*}$. Тогда в зависимости от известной исходной информации и ее объема возможно с определенной вероятностью формирование легальных сообщений. Для некоторых режимов блочного шифрования при некоторых параметрах режима эта вероятность равна единице.

Поэтому приведенная в ГОСТ $\mathrm{P} \quad 34.13-2015$ рекомендация ([1, стр. 24]) не использовать ключ имитозащиты в качестве ключа шифрования $\left(K \neq K^{*}\right)$ является существенной.

В случае когда режим выработки имитовставки реализуется как имитозащита шифрованных данных, для всех режимов шифрования, которые используют синхропосылку, возможно формирование легальных сообщений с вероятностью единица даже без знания открытых текстов.

Поэтому описанный в ГОСТ Р 34.13-2015 режим выработки имитовставки нельзя использовать как имитозащиту шифрованных данных.

\section{Список литературы}

[1] ГОСТ Р 34.13-2015. Криптографическая защита информации. Режимь работь блочных шифров, М. : Стандартинформ, 2015, 42 с.

[2] Гусев В.В., “Легальные сообщения для некоторых функций имитозащиты блочных шифров", Математические вопросы криптографии, 9:1 (2018), 27-46.

[3] Матюхин Д.В., Шишкин В.А., "Некоторые методы анализа функций хэширования и их применение к алгоритму ГОСТ Р 34.11-94", Математические вопросы криптографuu, 3:1 (2012), 71-90.

[4] Лось А.Б., Нестеренко А.Ю., Рожков М.И., Криптографические методы защиты информации : Учебник для академического бакалавриата, 2-е изд., испр., М. : Юрайт, 2016, 473 c. 
